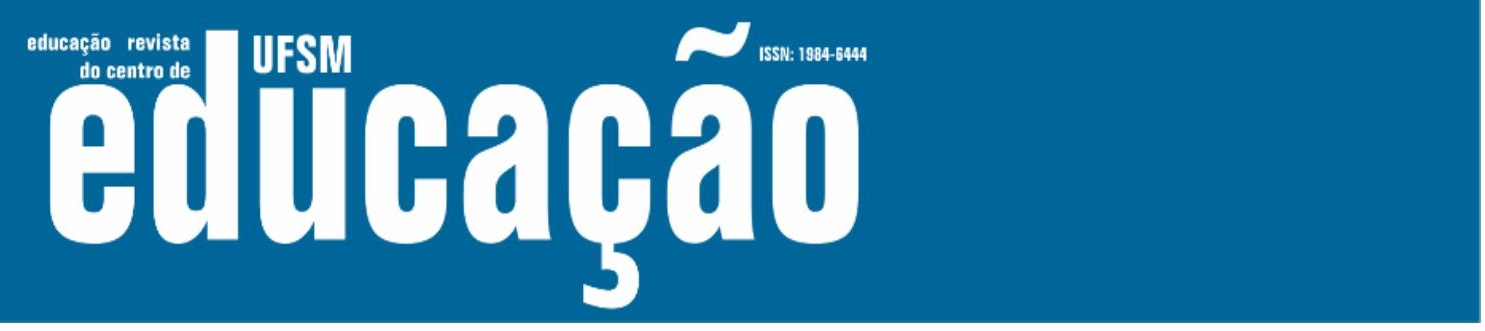

ISSN: 1984-6444 | http://dx.doi.org/10.5902/1984644439257

\title{
Dialogue Interculturel dans le curriculum pour la realisation de l'Education aux Droits de l'Homme: possibilites et limites
}

Intercultural dialogue in the curriculum for the realization of Human Rights Education: possibilities and limits

Diálogo Intercultural no currículo para a efetivação da Educação em Direitos Humanos: possibilidades e limites

Ana Maria Eyng

Professora doutora na Pontifícia Universidade Católica do Paraná, Curitiba, Paraná, Brasil. eyng.anamaria@gmail.com - http://orcid.org/0000-0003-0224-5880

Jéssica Adriane Pianezzola da Silva

Professora doutora na Pontifícia Universidade Católica do Paraná, Curitiba, Paraná, Brasil. jessicaadrianee@hotmail.com - https://orcid.org/0000-0001-9521-6725

João Casqueira Cardoso

Professor doutor na Universidade Fernando Pessoa, Porto, Portugal. jcasq@ufp.edu.pt - https://orcid.org/0000-0002-0894-452X

Recebido em 29 de julho de 2019

Aprovado em 03 de setembro de 2019

Publicado em 31 de janeiro de 2021

\section{RÈSUMÈ}

L'Éducation aux Droits de l'Homme est au centre de la problématique de cet article, compte tenu de la relation dialogique entre les politiques éducatives, les curricula et les perceptions des élèves. La méthode dialectique a guidé cette recherche, s'appuyant sur une approche qualitative, et inclue une recherche empirique intégrant la collecte des perceptions de plus de quatre cents élèves du secondaire et l'analyse des documents des politiques publiques et de neufs projets pédagogiques d'écoles secondaires brésiliennes. L'analyse des documents et des perceptions des jeunes permet d'identifier la fragilité de l'apprentissage et de l'expérience des Droits de l'Homme dans les écoles brésiliennes. Les élèves démontrent leurs attentes en matière d'Éducation aux Droits de l'Homme, exigeant un plus grand espace pour la participation, l'égalité de traitement, la liberté d'expression et le questionnement. L'Éducation aux Droits de l'Homme dans le cursus permettrait par ailleurs un dialogue entre l'égalité et la différence dans la perspective interculturelle critique.

Mots-clés: Droits de l'Homme; Curriculum scolaire; Interculturalité. 


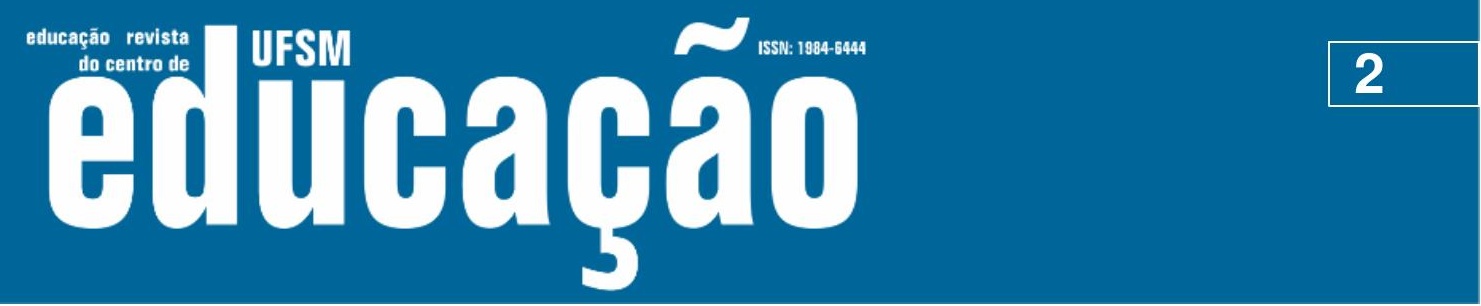

ISSN: 1984-6444 | http://dx.doi.org/10.5902/1984644439257

\section{ABSTRACT}

Human Rights Education is at the heart of the problematic of this article, given the dialogic relationship between the educational policies, curricula and perceptions of students. The dialectical method guided this research, using a qualitative approach, and included empirical research integrating the collection of perceptions more than four hundred high school students and the review of public policy documents and nine high school educational projects. The analysis of documents and perceptions of young people helps identify the fragility of learning and experience of Human Rights in Brazilian schools. Students demonstrate their expectations of Human Rights Education, requiring greater space for participation, equal treatment, freedom of expression and questioning. Human Rights Education in the curriculum would also allow a dialogue between equality and difference in the critical intercultural perspective. Keywords: Human Rights; School curriculum; Interculturality.

\section{RESUMO}

A Educação em Direitos Humanos está no cerne da problemática deste artigo, dada a relação dialógica entre as políticas educacionais, currículos e percepções dos estudantes. O método dialético orientou essa pesquisa, com base em uma abordagem qualitativa que incluiu pesquisas empíricas, integrando a escuta de percepções de mais de quatrocentos estudantes do ensino médio e a análise de documentos de políticas públicas e projetos pedagógicos de nove escolas de educação básica. $A$ análise de documentos e percepções de jovens ajuda a identificar a fragilidade da aprendizagem e da experiência dos Direitos Humanos nas escolas brasileiras. Os alunos demonstram suas expectativas em relação à Educação em Direitos Humanos, exigindo maior espaço para participação, tratamento igualitário, liberdade de expressão e questionamento. A Educação em Direitos Humanos no currículo também permitiria um diálogo entre igualdade e diferença na perspectiva crítica intercultural.

Palavras-chave: Direitos Humanos; Currículo escolar; Interculturalidade.

\section{Introduction}

La persistance de la violence engendrée par l'inégalité, l'injustice et l'exclusion sociale accompagne malheureusement encore la trajectoire de l'humanité. Dans l'histoire contemporaine, au cours des soixante-dix dernières années, nous avons été témoins des efforts internationaux en vue d'affirmer la dignité de tous et de toutes en termes de droits de l'Homme. Cependant, nous observons la nécessité de convertir ces droits en possibilités concrètes, parce que les droits de l'homme sont encore 


\section{OF HEM

ISSN: 1984-6444 | http://dx.doi.org/10.5902/1984644439257

soit comme une pure forme de citations des législations éducatives, et en somme une obéissance à une exigence légale, bien plus que la définition d'une véritable proposition d'EdH.

Tableau 1 - Incidence des droits de l'Homme dans les projets éducatifs

\begin{tabular}{lcccccccccc}
\hline \multirow{2}{*}{ CATÉGORIES IDENTIFIÉES } & \multicolumn{8}{c}{ NOMBRE DE MENTIONS PAR CATEGORIE ET } & \multirow{2}{*}{$\%$} \\
\cline { 2 - 11 } & $\mathbf{A}$ & $\mathbf{B}$ & $\mathbf{C}$ & $\mathbf{D}$ & $\mathbf{E}$ & $\mathbf{F}$ & $\mathbf{G}$ & $\mathbf{H}$ & $\mathbf{I}$ & \\
\hline Droits fondamentaux & 1 & 2 & 2 & 5 & 4 & 1 & 5 & 3 & 1 & $35,80 \%$ \\
\hline Contenus & 4 & 2 & 1 & 4 & - & - & 2 & - & 2 & $22,40 \%$ \\
\hline Droits citoyens & 5 & 3 & 1 & 1 & 1 & 1 & - & - & - & $17,90 \%$ \\
\hline Inclusion de la diversité & - & 2 & - & 2 & 1 & - & 2 & 1 & 1 & $13,40 \%$ \\
\hline $\begin{array}{l}\text { Lutte contre l'inégalité et la } \\
\text { violence }\end{array}$ & 2 & 1 & - & - & - & 1 & 1 & 1 & 1 & $10,40 \%$ \\
\hline TOTAL & $\mathbf{1 2}$ & $\mathbf{1 0}$ & $\mathbf{4}$ & $\mathbf{1 2}$ & $\mathbf{6}$ & $\mathbf{3}$ & $\mathbf{1 0}$ & $\mathbf{5}$ & $\mathbf{5}$ & $\mathbf{1 0 0 \%}$ \\
\hline
\end{tabular}

Source: Élaboré par les auteurs à partir des données de l'étude empirique.

En termes généraux, les documents analysés, bien qu'ils présentent certaines possibilités pour l'EdH, sont dans leur ensemble plus des limites que des adjuvants, parce que, bien que les droits de l'Homme y soient cités, ceux-ci ne sont pas assumés ou maintenus tout au long des projets. Ainsi, la possibilité d'être converti en actions pédagogiques et pratiques de coexistence dans la formation des élèves devient improbable.

En ce sens, pour faire la lumière sur la réalisation de l'EdH, nous examinons les perceptions des élèves qui fréquentent les écoles à partir desquelles nous analysons les projets pédagogiques. L'écoute des perceptions juvéniles peut apporter des éléments sur les processus de formation qu'ils expérimentent dans leurs écoles quand ils répondent à ce qu'ils comprennent pour les droits de l'Homme à l'école et sur l'insertion du thème dans les curricula de leurs écoles. Les deux questions, sur ces deux domaines (ce en quoi consistent les droits de l'Homme; et la place qui leur est réservée dans les curricula) ont été posées en deux étapes, initialement sous la forme d'une question ouverte et dans un second temps sous la forme d'une question fermée. 


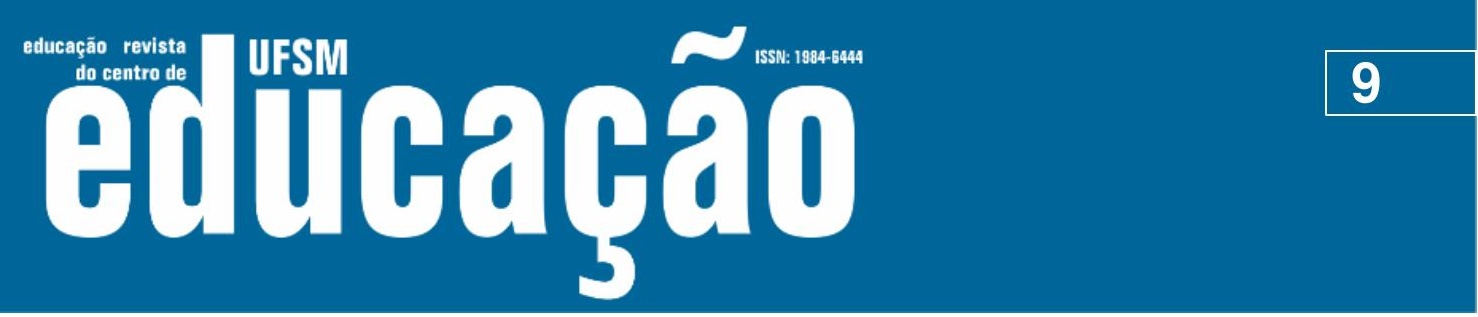

ISSN: 1984-6444 | http://dx.doi.org/10.5902/1984644439257

I - par transversalité, au moyen de sujets liés aux droits de l'Homme et traités de manière interdisciplinaire; II - en tant que contenu spécifique d'une des disciplines déjà existantes dans le curriculum scolaire; III - de manière mixte, c'est-à-dire combinant la transversalité et l'approche par disciplines (BRÉSIL, 2012, art. 6ème).

La résolution ajoute, en un paragraphe unique, une remarque que nous considérons comme très générique et qui peut être convertie davantage en une limite qu'en une possibilité de mettre en œuvre l'EdH dans le curriculum.

D'autres formes d'insertion de l'éducation aux droits de l'Homme peuvent encore être admises dans l'organisation curriculaire des établissements d'enseignement, à condition que les spécificités des niveaux et des modalités de l'éducation nationale soient respectées» (BRÉSIL, 2012, art. 7ème).

II y a donc une contradiction dans la résolution, qui présente d'un côté la transversalité comme principe d'EdH, et comme un moyen d'organiser la planification, les matériaux et les stratégies, mais qui à l'article 7ème présente des travaux ponctuels dans une discipline ou un contenu comme une possibilité, et ouvrent en outre l'éventualité d'autres possibilités.

Tableau 2 - Approches des droits de l'Homme dans les curricula

\begin{tabular}{|l|}
\hline I. ALTERNATIVES DE RÉPONSES \\
\hline 1. Intégré - Approche «transversale» dans toutes les disciplines du secondaire, à travers des \\
sujets liés aux droits de l'Homme, à la justice et à la coexistence \\
\hline 2. Par matière - Approche à travers une matière spécifique à insérer dans le curricula. \\
\hline 3. En alternance - Approche mixte, c'est-à-dire interdisciplinaire et par matière. \\
\hline $\begin{array}{l}\text { 4. Thèmes spécifiques - Traitement comme contenu spécifique d'une ou plusieurs matières déjà } \\
\text { existantes dans le programme des études secondaires. }\end{array}$ \\
\hline 5. Application de principes - Dans le contexte professionnel et dans la formation continue. \\
\hline 6. Vie quotidienne - Vie à l'école orientée vers les questions des droits et de la Justice. \\
\hline 7. Aucun besoin d'insérer dans le programme des études secondaires. \\
\hline
\end{tabular}

Source: Élaboré par les auteurs à partir des données de l'étude empirique.

Les perceptions exprimées par les élèves ont permis d'extraire les catégories suivantes, par ordre d'appréciation: 1. Application des principes; 2. Intégré; 3. Par matière; 4. Vie quotidienne; 5. Sujets spécifiques; 6. Autre type de travail; 7. Pas nécessaire. 


\section{usm

ISSN: 1984-6444 | http://dx.doi.org/10.5902/1984644439257

Le positionnement des élèves en ce qui concerne l'insertion de l'EdH dans le programmes d'études peut être mieux compris en rapportant à celui-ci leur perception des droits de l'Homme à l'école. En répondant à la question ouverte sur ce qu'ils entendent par les droits de l'Homme à l'école (première étape), les élèves ont indiqué à la fois leurs attentes de droits que l'espace scolaire peut permettre, ainsi que les conditions nécessaires en vue de leurs concrétisations.

Les perceptions exprimées par les étudiants dans la première phase de la recherche, composé pour questions ouvertes, ont permis l'extraction des catégories qui ont préparé le terrain de la deuxième phase, qui inclue des questions fermées. Dans la préparation du formulaire appliqué dans la deuxième phase de la recherche, l'ensemble des réponses des élèves ont été regroupées au sein des options indiquées au Tableau 3, suivies d'une brève explication, qui résulte des réponses des participants lors de la première phase. On a demandé aux élèves de selectionner la pertinence d'un certain nombre d'options relatives aux droits de l'Homme dans les écoles. 


\section{تilloapẫ}

ISSN: 1984-6444 | http://dx.doi.org/10.5902/1984644439257

invités à choisir parmi les treize catégories présentées, indiquant laquelle parmi cellesci correspond aux meilleures alternatives en vue de définir les droits de l'Homme à l'école. Dans le Tableau 4, les choix des définitions des droits de l'Homme à l'école sont présentés dans l'ordre d'importance qui leur a été attribuée par les élèves.

Tableau 4 - Perceptions dominantes sur les droits de l'Homme à l'école - seconde phase

\begin{tabular}{l|c|c}
\hline ORDRE DE LA CLASSIFICATION - DROITS DE L'HOMME A L'ECOLE & FRÉQ. & $\%$ \\
\hline Droit à la liberté d'expression & 65 & 14,7 \\
\hline Droit à une éducation de qualité & 62 & 14 \\
\hline Droit à l'égalité & 61 & 13,8 \\
\hline Détermination, normation morale & 47 & 10,6 \\
\hline Droit à la différence & 33 & 7,5 \\
\hline Droit au respect & 30 & 6,8 \\
\hline Droit à la convivialité & 28 & 6,3 \\
\hline Droit à jouer un rôle & 19 & 4,3 \\
\hline Droit à l'accès l'école & 18 & 4,1 \\
\hline Droit au développement intégral & 17 & 3,8 \\
\hline Droit à la protection & 16 & 3,6 \\
\hline Droit à l'intégrité & 10 & 2,3 \\
\hline Droit à l'inclusion & 0 & 0 \\
\hline Autres & 1 & 0,2 \\
\hline Sans réponse & 37 & 8,4 \\
\hline Total & 443 & 100 \\
\hline
\end{tabular}

Source: Élaboré par les auteurs à partir des données de l'étude empirique.

Les choix des élèves décrivent à la fois leurs croyances sur ce qu'elles ou ils comprennent sur la garantie des droits de l'Homme. Par ailleurs, ils attestent ce que l'on peut dénommer le déni de la mission de concrétisation des droits de l'Homme, voire leur violation, au sein même de certaines écoles publiques au Brésil. Les données recueillies apportent de nombreux éléments afin de repenser l'insertion de l'EdH, guidée par des conceptions et des pratiques interculturelles de garantie des droits.

Les résultats que nous avons signalés indiquent plus particulièrement trois points: d'abord, le point culminant des politiques éducatives pour la garantie du droit à 


\section{Eulfoapẫ}

ISSN: 1984-6444 | http://dx.doi.org/10.5902/1984644439257

l'éducation, ensuite, le manque de respect pour l'insertion obligatoire de l'EdH dans une grande partie des projets des écoles analysées, enfin, la perception des élèves sur les droits de l'Homme à l'école valorise le droit à la liberté d'expression en tant que droit de questionner, de s'exprimer librement et de participer; le droit à une éducation de qualité, considéré aussi bien quant aux enseignants qu'aux infrastructure adéquates; et le droit à l'égalité, par l'égalité de traitement sans distinction ni discrimination.

Dans ces points, nous pouvons identifier les possibilités et les limites qui constituent de grandes contradictions qui doivent être envisagées et surmontées afin que l'EdH puisse être efficace dans les programmes scolaires.

Une grande potentialité pour l'EdH, lorsque nous observons l'accent mis sur les politiques éducatives pour garantir le droit à l'éducation, considéré comme un droit fondamental, dont les autres droits peuvent être conquis et pour le droit à la diversité dans Curriculum, indépendamment de la race, l'ethnicité, le sexe, la culture, la sexualité, etc; En revanche, la contradiction qui est configurée comme une limite, est dans la présence encore très commune des discours et des pratiques curriculaires fondées sur des hypothèses conservatrices, hégémoniques et monoculturelles, médiée par des méthodologies conteudistes, des évaluations Standardisé et meritrocratique, par conséquent, excludent.

Une grande fragilité pour l'EdH, lorsque nous observons le manque de respect de l'inclusion obligatoire de l'EdH dans une grande partie des projets curriculaires analysés, qui quand ils insèrent le thème le faire sur une base ponctuelle, sans base suffisante pour soutenir la formation dans Pour et avec $\mathrm{DH}$.

Une autre potentialité est révélée dans les perceptions des jeunes sur les droits de l'homme à l'école, en valorant le droit à la liberté d'expression, en tant que droit à la question, à s'exprimer librement et à participer. Le droit à une éducation de qualité, considéré comme une condition pour garantir une éducation de qualité, des enseignants et des infrastructures adéquates. Le droit à l'égalité en tant que traitement égalitaire sans distinction ni discrimination. Cependant, les attentes des droits exprimés dans les perceptions des élèves n'ont pas été garanties dans l'école quotidienne, les transformant en frustrations, et par conséquent, en créant des limites 


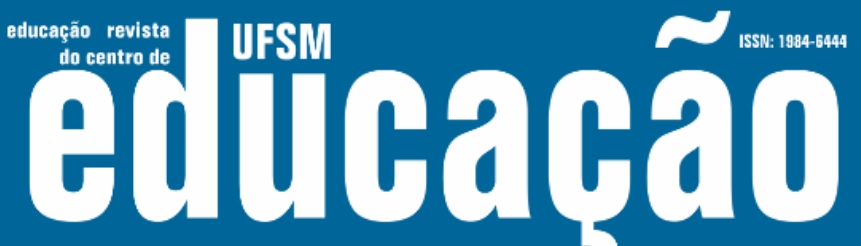

ISSN: 1984-6444 | http://dx.doi.org/10.5902/1984644439257

l'intégration de tous les espaces, langues, savoir-faire et acteurs impliqués dans les processus éducatifs.

En transformant l'espace scolaire en espace de garantie et d'expérience effective des droits de tous et de toutes, reconnaissant l'éducation en tant que droit social effectif, il conduit aux sujets eux-mêmes, aux occasions de connaître et de vivre dans des contextes qui respectent la diversité. Dans ces contextes, le dialogue peut être initié par la reconnaissance et la discussion des lieux partagés entre les cultures, dans le processus que Boaventura de Sousa Santos (2010) nomme l' «herméneutique diatopique», où l'on parvient collectivement à un consensus plus inclusif, soulignant des valeurs telles que le respect, la justice, et l'équité.

Là encore, nous insistons sur le rôle fondamental de la participation des élèves. Toutefois, seul un éducateur formé à l'interculturalité peut donner la parole aux élèves et reconnaître l'incidence des différentes conceptions culturelles qui sont présentées dans les curricula et les programmes scolaires, à travers la voie des discours globalisants et éventuellement hégémoniques. Le dévoilement de ces discours est également une étape importante dans la déconstruction des récits de subordination qui ont conduit à la fausse reconnaissance et au non respect de la diversité culturelle, car, en incorporant des différences, ils tendent à les neutraliser et à les vider de leur signification (WALSH, 2009). Les éducateurs et les élèves peuvent ainsi servir de médiateur et participer au dialogue interculturel nécessaire en $\mathrm{EdH}$ dans les curricula scolaire, en contribuant à la reconnaissance de processus intégratifs susceptibles de renforcer les contributions des diverses cultures. Dans ce processus de médiation et de participation, il est essentiel d'identifier et de surmonter les contradictions politiques et pratiques qui deviennent des limites pour l'efficacité des EdH dans le cursus, et qui en sont en fait le principal obstacle.

\section{Considérations finales}

Dans cette étude, en inscrivant à l'ordre du jour l'analyse des politiques publiques, les curricula scolaires et des perceptions des élèves, nous avons tenté de dévoiler les exigences du dialogue interculturel pour l'effectivité de l'Éducation aux 


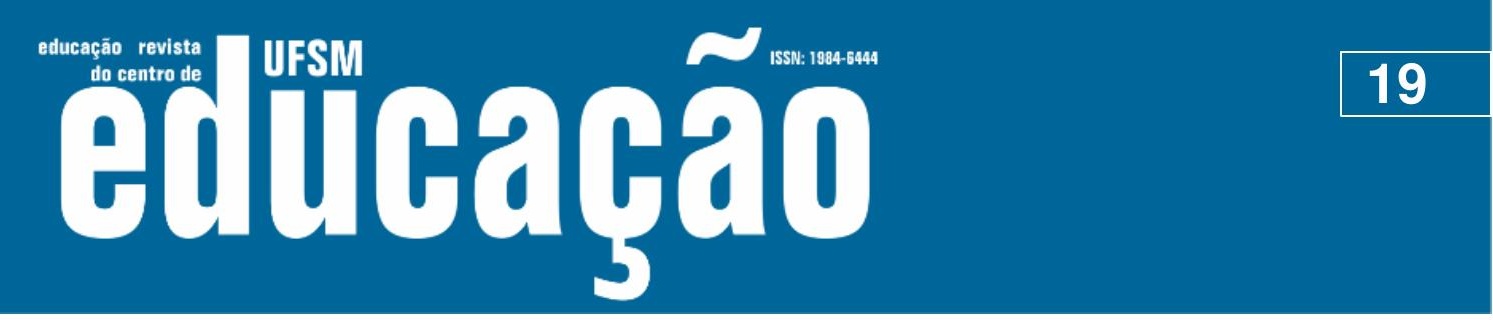

ISSN: 1984-6444 | http://dx.doi.org/10.5902/1984644439257

là une fausse promesse faite à un énorme contingent de la population mondiale, plus particulièrement concentré en Amérique latine et en Afrique, auquel aucune conversion des propositions de politique des droits de l'Homme ne parvient à se transformer en actions concrètes. En ce sens, d'assumer l'interculturalité comme un principe et une méthode pédagogique dans la formulation et l'exécution effective des politiques publiques promeut les droits contre l'hégémonie, préconise d'autres sensibilités, d'autres façons d'être dans le monde et de nouvelles sociabilités, soucieuses de surmonter les inégalités et les processus d'exclusion qui persistent encore dans les sociétés contemporaines.

\section{Références bibliographiques}

ARROYO, Miguel Gonzales. Currículo, território em disputa. 2. ed. Petrópolis: Vozes, 2011.

BARDIN, Laurence. Análise de Conteúdo. Lisboa: Edições 70, 1996.

BRÉSIL. [Constitution (1988)]. Constituição da República Federativa do Brasil. Brasília, DF: Congresso Nacional, 1988.

BRÉSIL. Decreto n. 7.037, de 21 de dezembro de 2009. Aprova o Programa Nacional de Direitos Humanos - PNDH-3 e dá outras providências. Diário Oficial da União, Brasília, DF, seção 1, p.17, 22 dez. 2009.

BRÉSIL. Plano Nacional de Educação em Direitos Humanos. Brasília, DF: SEDH/MEC/MJ/UNESCO/CNEDH, 2007.

BRÉSIL. Resolução MEC/CNE n. 01, de 30 de maio de 2012. Institui Diretrizes Nacionais para a Educação em Direitos Humanos. Diário Oficial da União, Brasília, DF, seção 1, p. 33, 30 maio 2012.

CANDAU, Vera Maria Ferrão et al. Educação em direitos humanos e formação de professores(as). São Paulo: Cortez, 2013.

CANDAU, Vera Maria Ferrão. Direitos Humanos, educação e interculturalidade: as tensões entre igualdade e diferença. Revista Brasileira de Educação, v. 13, n. 37, p. 45-56, 2008.

CARDOSO Jr, Wilson; CANDAU, Vera Maria Ferrão. Interculturalidade e ensino de artes visuais do Colégio Pedro II. Educação, Santa Maria, v. 43, n. 4, p. 721-740, out./dez. 2018. 


\section{usm \\ 〜 m.mum

ISSN: 1984-6444 | http://dx.doi.org/10.5902/1984644439257

ESPEJO, Juan Rolando Cornejo. Educación, interculturalidade y cidadania. Educar em Revista, v. 28, n. 43, p. 239-254, jan./mar. 2012.

ONU [Organização das Nações Unidas]. Conferência Mundial de Viena sobre os Direitos Humanos. Viena: Assembleia Geral da ONU.n.43, p. 239-254, 1993.

ONU [Organização das Nações Unidas]. Declaração Universal dos Direitos Humanos. New York: Assembleia Geral da ONU, 1948.

SANTOS, Boaventura de Sousa. A gramática do tempo: para uma nova cultura política. São Paulo: Cortez, 2010.

SANTOS, Boaventura de Sousa. Para uma sociologia das ausências e uma sociologia das emergências. Revista Crítica de Ciências Sociais, v. 63, p. 237-280, out. 2002.

TAPIAS, José Antônio Pérez. Educar a partir da interculturalidade: exigências curriculares para o diálogo entre culturas. In: SACRISTÁN, José Gimeno (org.). Saberes e incertezas sobre o currículo. Porto Alegre: Penso, 2013.

UNESCO [Organisation des Nations Unies pour l'Éducation, la Science et la Culture]. Programa Mundial para Educação em Direitos Humanos. Plano de Ação: Primeira Fase. 2005.

UNESCO [Organisation des Nations Unies pour l'Éducation, la Science et la Culture]. Programa Mundial para Educação em Direitos Humanos. Plano de Ação: Segunda Fase. 2012.

UNESCO [Organisation des Nations Unies pour l'Éducation, la Science et la Culture]. Programa Mundial para Educação em Direitos Humanos. Plano de Ação: Terceira Fase. 2015.

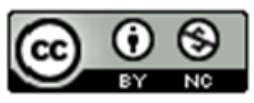

This work is licensed under a Creative Commons Attribution-NonCommercial 4.0 International (CC BY-NC 4.0) 